\title{
Motor pattern of the left colon before and after surgery for rectal cancer: possible implications in other disorders
}

\author{
B N CATCHPOLE \\ From the Departments of Surgery, St Mark's Hospital, London, and the University of Western Australia, Queen \\ Elizabeth II Medical Centre, Nedlands, Western Australia
}

SUMmary The motility of the descending colon was studied in nine patients before and after resection of the rectum for cancer. This operation parasympathectomises and generally also sympathectomises the remaining left colon. Motor activity was significantly reduced by sleep before and after surgery. There was also a reduction in awake motility after surgery which is attributed to extrinsic denervation. The reduced motility may explain the increased frequency of motions passed after surgery.

It is a common observation that after restorative resection of the rectum for cancer, the patient's bowel emptying pattern is often disturbed for a period of months. In fact, it may never return to the preoperative state.

Amongst possible causes, the loss of local sensing reflexes and of a dehydrating and storage segment of the gut are obvious. What is often not appreciated is that resection of the rectum removes the parasympathetic and often also the sympathetic nerve supply to the remaining left colon. The parasympathetic supply to the distal colon arises on each side from the inferior hypogastric plexus. The nerves pass upwards towards the distal transverse colon, across the sigmoid vessels and the branches of the inferior mesenteric artery; some fibres run on the bowel wall. Subdivisions of the nerves continue to the splenic flexure. Thus the nerves sandwich the mesenteric arteries. ${ }^{12}$ Only rarely are some fibres distributed along the branches of the inferior mesenteric artery, which is the usual route for the sympathetic nerves to follow.

Resection of the rectum or of sigmoid colon with section of the mesentery will generally therefore parasympathetically denervate the distal third of the

Address for correspondence: Professor B N Catchpole, University Dept of Surgery, Queen Elizabeth II Medical Centre. Nedlands, W Australia 6())9.

Received for publication 5 November 1987. transverse colon and that beyond it down to the anastomosis - or iliac colostomy. If the inferior mesenteric pedicle is divided also, a similar length of colon is probably separated from its sympathetic nerve supply. Such denervations are virtually constant in the radical resection of the rectum with its lymphatic territory but their consequences have received little if any examination.

This study was made, therefore, in the first place to investigate the effects of resection of the extrinsic nervous innervation of the left colon as a consequence of rectal resection and to endeavour to relate any findings to postoperative bowel function. In the second place, it was thought possible that the study might provide an indication as to whether extrinsic denervation could have potential in the treatment of motor disorders of the colon such as diverticulosis, which is believed to be the result of areas of mucous membrane being driven through the muscle wall by intraluminal pressure. ${ }^{3}$

The investigation was approved by the Human Rights Committee of St Bartholomew's Hospital, London.

\section{Methods}

PATIENTS

Nine patients (five men) aged between 47 and 75 years were studied before radical resection for 
carcinoma of the rectum and at least two months after surgery. All had lesions which permitted the passage of a colonoscope into the descending colon. The patients were otherwise well and none had diabetes mellitus, systemic nervous disease, or colonic diverticulosis.

Each patient had the objects and details of the investigation explained to him/her and eàch agreed to return to hospital for one night after recovery from surgery for the postoperative part of the study.

Recordings were made on all occasions from the early evening through to the next morning after breakfast in order to obtain a waking and sleeping pattern of left colonic motility.

A detailed history was obtained of each patient's lifestyle, eating and bowel habits before the onset of the symptoms of the rectal neoplasm as well as details of the lesion's effect on these features. On return for the second recording, postoperative eating habits, bowel habit, and its effect on the patient's lifestyle were again recorded.

The motor pattern of the left colon was recorded by an intraluminal triple miniature balloon $(2 \mathrm{ml}$ each) catheter system. The balloons, made of thin rubber, were $8 \mathrm{~cm}$ apart and sited on a non-compliant catheter, stiffened by the incorporation of an angiographic guide wire; this prevented the tube from dislodging readily and made radiographic localisation easy. The water filled balloon system recorded via individual Statham transducers on a Grass polygraph*. Respiration was coincidentally monitored by a mercury in silastic rubber strain gauge comfortably located round the abdomen at umbilical level. This permitted not only respiration but other movements affecting the abdomen to be recorded. The polygraph tracings were made on paper moving at $15 \mathrm{~mm} /$ minute and were continuous for not less than 12 hours overnight during which the patient took at least one meal.

The recording balloon section of the catheter system was located in the descending colon. The patient's colon was prepared by two phosphate enemas but no general sedation was given. A colonoscope was passed to the desired position in the colon towing the balloon catheter system with it. This was achieved by tying a thread to the tip of the system and drawing this down the biopsy channel of the fibrescope. The thread was then fixed to the handpiece of the instrument so that the tip was just behind the viewing end. When appropriately positioned, the thread was cut at the handpiece and the colonoscope withdrawn leaving the catheter in situ. It was securely taped to the skin as it emerged from the anus.

The patient returned to bed and remained there

${ }^{*}$ Grass Instrument Company, Quincy, Mass, USA. until the end of the recording. No patient wanted to defecate during the period of the recording - the left bowel being largely empty. Micturition was either into a bedpan or urinal. Meals and liquids were taken without any modification of normal timing and at the patient's desire, but were recorded so that correlation with bowel motor events could be made. The times of lying down to sleep and of awakening were also noted. Drugs were avoided if at all possible for the 24 hours before and during the period of study.

After the night's recording, the catheter system was located by radiograph. It rarely moved markedly, although on some occasions it retreated to the proximal sigmoid colon in the initial study.

After full recovery from surgery and at least two months after operation, each patient was re-admitted to hospital and the second overnight recording carried out. The sensing balloons of the triple lumen system were located as accurately as possible in the same segment of gut as they had been at the first recording. Also, the same bowel preparation and colonoscopic placement method was used as in the first study. Again no premedication was used.

Of the nine patients studied, all were presumed to have had the remaining part of their hindgut parasympathectomised. Seven patients were also presumed to have lost sympathetic innervation to the hindgut as the inferior mesenteric artery was divided on the aorta. In two patients, the remaining hindgut was probably not sympathectomised as the inferior mesenteric artery was tied just below its highest branch. Two patients had terminal iliac colostomies.

\section{ANALYSIS OF RECORDS}

The records were analysed by hand. Five specific periods were studied in each trace. These were: 600-700 pm early evening; $900-1000 \mathrm{pm}$ late evening; 1030-1230 am early sleep; 600-700 am waking; 800-900 am postbreakfast.

General observations were made from the whole of each recording. Variables were measured in each of the periods noted above:

\section{(a) Total activity (TA)}

For all of the specific periods considered, the percentage times that each of the three traces were above their individual baselines, were calculated. Total activity is the mean of these three values.

\section{(b) Motility index (MI)}

A planimeter was used to measure in arbitrary units, the surface area under the traces. The motility index presented in each case is the mean value obtained from the traces of the three channels over 15 minutes in the sleeping phase just before waking and on waking. 
Table 1 Patients' bowel habits (motions/day) before and after surgery

\begin{tabular}{|c|c|c|c|c|c|}
\hline Patient & $\begin{array}{l}\text { Lifelong } \\
\text { habit }\end{array}$ & $\begin{array}{l}\text { Frequency } \\
\text { at } \\
\text { study I }\end{array}$ & $\begin{array}{l}\text { Frequency } \\
\text { at return } \\
\text { home }\end{array}$ & $\begin{array}{l}\text { Frequency } \\
\text { at } \\
\text { study II }\end{array}$ & $\begin{array}{l}\text { Time to } \\
\text { postop. } \\
\text { study } \\
\text { (weeks) }\end{array}$ \\
\hline 1 & 2 & $5-6^{*}$ & $2^{*}$ & 2 & 17 \\
\hline 2 & 1 & 2 & $4-5$ & 1 & 30 \\
\hline 3 & 1 & $2-3$ & $3-4$ & 2 & 17 \\
\hline 4 & $1-2$ & 4 & Fluid (C) & 1 only $(C)$ & 15 \\
\hline 5 & $\begin{array}{l}\text { Only with } \\
\text { laxative }\end{array}$ & 3 & $\begin{array}{l}\text { Semi } \\
\quad \text { fluid (C) }\end{array}$ & am only (C) & 9 \\
\hline 6 & 1 & 1 & $4^{*}$ & 2 & 18 \\
\hline 7 & 1 & 3 & $24^{*}$ & $6-7$ & 18 \\
\hline 8 & 3 & $4-5$ & $3-4^{*}$ & $2-3$ & 17 \\
\hline 9 & $\begin{array}{l}\text { 1/3 days } \\
\text { with } \\
\text { laxative }\end{array}$ & $\begin{array}{l}\text { 1/3 days } \\
\text { with } \\
\text { laxative }\end{array}$ & $6^{*}$ & $5-6$ & 14 \\
\hline
\end{tabular}

$\mathrm{C}=$ colostomy $;{ }^{*}=$ urgency of bowel habit making the patient house bound.

\section{(c) Maximum period of total inactivity (MPI)}

This is the maximum duration (in minutes) of total gut inactivity measured on each channel pre and postoperatively from the complete set of traces. This provided six observations per patient.

Differences of these variables during sleeping and waking, and the effects of operation were sought. The Wilcoxon's signed-rank test (two sided) was used in analysis of TA and MI. Maximum period of total inactivity was analysed using the $t$ test and the Hotellings $\mathrm{T}^{2}$ test.

\section{Results}

The recording technique was in general satisfactory. Two of the nine patients were given terminal left iliac colostomies; the postoperative recordings in these two patients were made via the colostomy, the recording system lying in the descending colon, from which recordings had been made in the preoperative study.

The individual patient's bowel habits are shown in Table 1. None of the patients showed any diverticulosis of the colon either in the preoperative investigation or in the operative specimen.

Bowel habit had in most patients increased as a result of their disease and in several, further increased postoperatively. The two colostomy patients passed fluid motions almost continuously in the first weeks after surgery. Five patients immediately after surgery were virtually confined to their homes for an initial few weeks owing to the unpredictability of the call to stool, its urgency, and the fear of faecal incontinence.
By the time of the second study, frequency was reducing but had not returned to the habitual pattern in five of the patients.

MOTILITY RECORDS

\section{General observations}

The predominant pattern was of major waves of less than 5 cycles/minute, although in some patients before and after surgery faster waves of over 5 cycles/minute and up to 12 cycles per minute occurred in relatively short bursts. The major waves generated the greatest pressure. In general there was little faster activity either pre or postoperatively. In some records, compound waves were observed. General observations from the records were that: (1) Contractile activity did not necessarily involve all channels at the same time although this was apparent on other occasions. (2) All left colon activity was greatly reduced during sleep, arousals and full awakening from sleep usually causing almost immediate resumption of diffuse activity (Fig. 1a) preoperatively. Decrease of activity after surgery was also noted during sleep. On waking, increased activity required several minutes to develop (Fig. 1c). (3) Similarly, the arrival of food for the patient increased activity on all channels (Fig. 1b) preoperatively, a phenomenon delayed by surgery (Fig. 1d). (4) Left colonic activity appeared to be generally reduced by extrinsic autonomic denervation - that is, in the second study compared with the first (Fig. 1a and $\mathrm{b} v$ Fig. 1c and d).

\section{TOTAL ACTIVITY}

The mean of the percentages of the nine patients' three channels for the pre and postoperative five periods are presented in Fig. 2, where it appears that activity in all periods is reduced by operation. When the pre and postoperative values of total activity are examined by the Wilcoxon's signed-rank test, however, the differences do not quite reach the conventional statistical significance level $(p=0 \cdot 063)$.

These data suggest that there may be a reduction as a result of surgery.

There is a highly statistically significant reduction in total activity during sleep compared with the awake period (Wilcoxon's signed-rank test: $p=0.008$ ) when the summed values of the $1030 \mathrm{pm}$ to $1230 \mathrm{am}$ periods pre and postoperatively of the nine patients are compared with the awake period at 6-7 am. Furthermore the differences in the preoperative values at the same times are significant $(p=0.004)$ as also are the postoperative ones $(p=0.036)$ suggesting the reduction is independent of the colon's nerve supply at the site studied. 

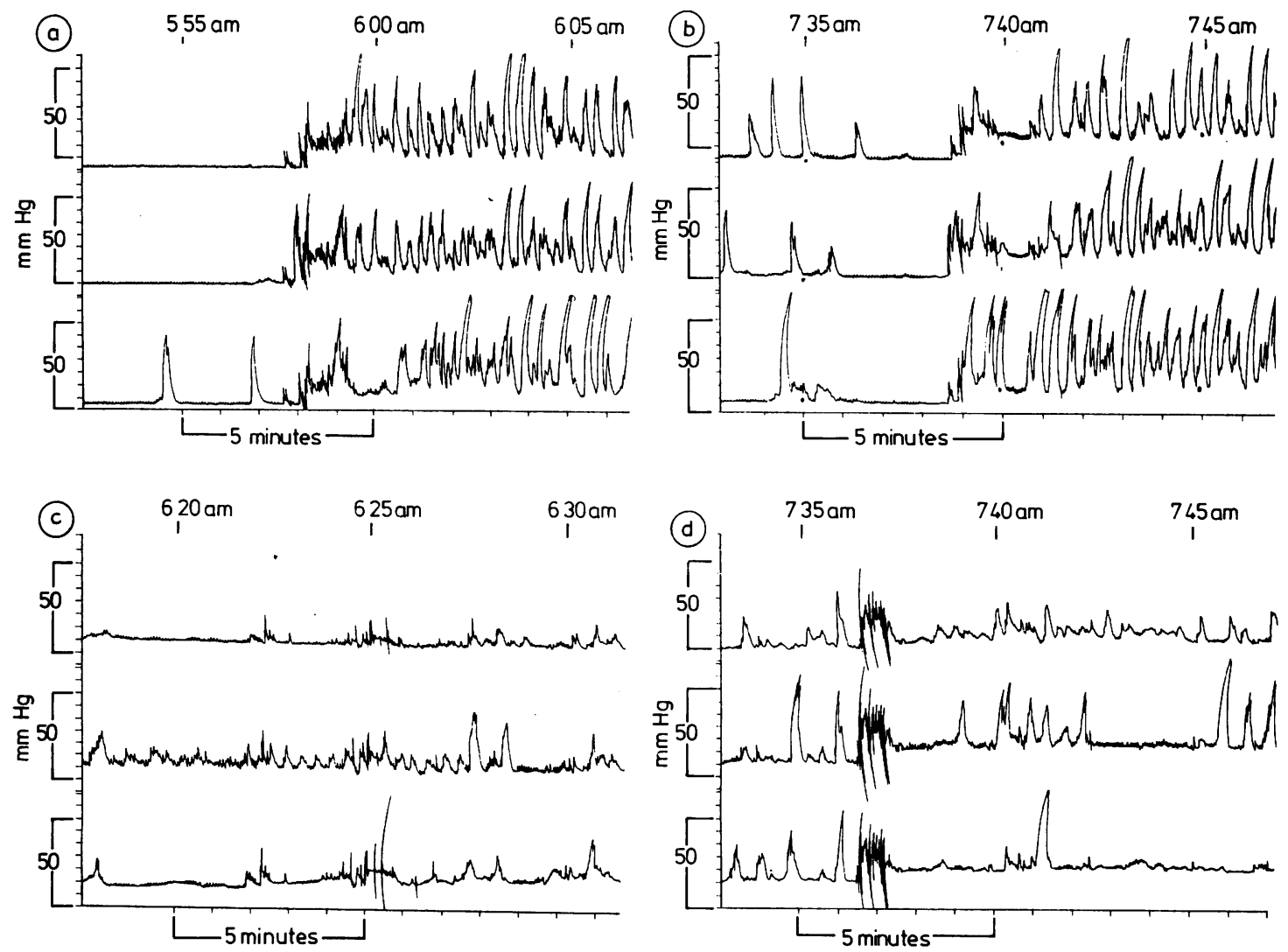

Fig. 1 (a) Preoperative recording showing low sleeping activity with rapid onset of three channel activity on waking at 558 am. (b) Preoperative recording from same patient at the time of arrival of his breakfast at 739 am. Baseline rose as the patient sat up in bed. (c) Postoperative recording from same patient as in Figures 1(a) and (b) on waking at 625 am. Immediate increase in activity did not occur. (d) Corresponding tracing to Fig. 1(b) at the time of the arrival of breakfast at 737 am. Pressures in most waves have also been apparently reduced by surgery. Baseline rose as the patient sat up in bed.

There was no apparent association between the means of total activity of the various time periods and the frequency of defaecation, either pre or postoperatively.

\section{MOTILITY INDEX (MI)}

The means of the 15 minute motility indices obtained immediately before and on waking from the available channels were obtained for each patient from the pre and postoperative traces (Table 2).

Comparison of the sleeping and awake values indicates greater activity after waking (Wilcoxon's test $\mathrm{p}=0 \cdot 02$ ).

Subtraction of the sum of the sleeping and awake indices obtained postoperatively from the sum of those obtained preoperatively indicates that there was a significant fall in the index after surgery (Wilcoxon's test: $p=0 \cdot 004)$. The two patients (nos 5 and 8) who were unsympathectomised did not have obviously different values.

MAXIMUM PERIODS OF TOTAL INACTIVITY (MPI) These are shown in Table 3 and it is apparent how variable in contractile activity are segments of the bowel adjacent to each other. In general it appeared that activity was reduced postoperatively when the sum of the preoperative values was compared with the sum of the postoperative values; however, a $t$ test to assess difference did not achieve statistical significance $(p \approx 0 \cdot 10)$ although there is a suggestion that postoperative inactivity is increased. If the results of the two patients retaining their sympathetic nerves are not included, post-operative inactivity appears to be significantly greater $(t$ test, $p<0.01)$ in the postoperative period.

There was no apparent relationship of the fre- 


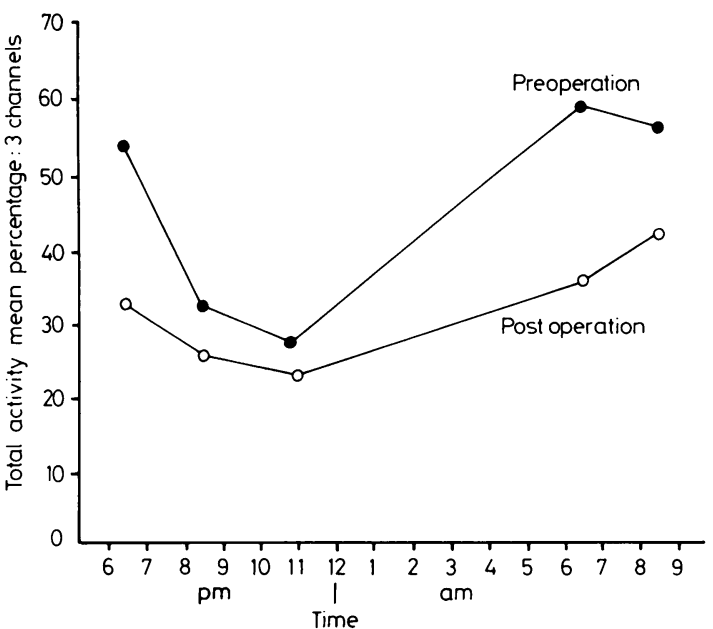

Fig. 2 Group shows mean percentages of total activity from the nine patients from the pre and postoperative recordings. Difference between these does not reach statistical significance $(p=0.063)$ but the reduction during sleep compared with the awake period is significant.

quency of bowel habit to the mean duration of inactivity recorded from the three channels.

\section{Discussion}

The understanding of the relationships of gut motility pattern to function is in its infancy. It has been shown that the 24 hour pattern of colonic motility may not be the same in the succeeding 24 hours and that preparation of the bowel also affects its pattern compared with that of the unprepared bowel. ${ }^{45} \mathrm{We}$ need to know for how long after enema preparation the altered motility response lasts. Another difficulty

Table 2 Motility indices obtained over 15 minutes immediately before and on awakening from patients' traces obtained pre and postoperatively

\begin{tabular}{|c|c|c|}
\hline \multirow[b]{3}{*}{ Patient } & Sleeping & Awake \\
\hline & Operation & Operation \\
\hline & Pre Post & Pre Post \\
\hline 1 & $14 \rightarrow 8$ & $28 \rightarrow 24$ \\
\hline 2 & $66 \rightarrow 9$ & $109 \rightarrow 47$ \\
\hline 3 & $15 \rightarrow 9$ & $116 \rightarrow 27$ \\
\hline 4 & $13 \rightarrow 18$ & $33 \rightarrow 11$ \\
\hline 5 & $36 \rightarrow 0$ & $137 \rightarrow 55$ \\
\hline 6 & $65 \rightarrow 0$ & $179 \rightarrow 100$ \\
\hline 7 & $5 \rightarrow 16$ & $264 \rightarrow 49$ \\
\hline 8 & $120 \rightarrow 10$ & $136 \rightarrow 100$ \\
\hline 9 & $30 \rightarrow 33$ & $29 \rightarrow 3$ \\
\hline
\end{tabular}

Table 3 Maximum periods of total inactivity in each channel recorded (in minutes) (a) in the preoperative and (b) in the postoperative study

\begin{tabular}{|c|c|c|c|}
\hline \multirow[b]{2}{*}{ Patient } & Channell & Channel 2 & Channel 3 \\
\hline & (a) (b) & (a) (b) & (a) (b) \\
\hline 1 & $15 \rightarrow 49$ & $6 \rightarrow 16$ & $24 \rightarrow 18$ \\
\hline 2 & $15 \rightarrow 12$ & $15 \rightarrow 47$ & $15 \rightarrow 65$ \\
\hline 3 & $28 \rightarrow 42$ & $36 \rightarrow 49$ & $28 \rightarrow-$ \\
\hline 4 & $36 \rightarrow 40$ & $35 \rightarrow 77$ & $39 \rightarrow 71$ \\
\hline $5^{*}$ & $33 \rightarrow 59$ & $71 \rightarrow 56$ & $34 \rightarrow 68$ \\
\hline 6 & $15 \rightarrow 149$ & $31 \rightarrow 51$ & $15 \rightarrow 107$ \\
\hline 7 & $25 \rightarrow 100$ & $25 \rightarrow 34$ & $18 \rightarrow 36$ \\
\hline $8^{*}$ & $28 \rightarrow 32$ & $99 \rightarrow 10$ & $66 \rightarrow 13$ \\
\hline 9 & $31 \rightarrow 34$ & $60 \rightarrow 102$ & $7 \rightarrow 9$ \\
\hline
\end{tabular}

Patients 5 and $8\left({ }^{*}\right)$ retained their sympathetic nerves to the segment studied.

in the interpretation of colonic motility in the first series of this study involves the potential effect of the rectal neoplasm's presence. The consequence of this was to increase the frequency of defecation of seven of the nine patients studied, but no patient defecated during the actual recording probably because the two phosphate enemas had emptied the left side of colon. When studies are eventually made of the right and left sides of the colon in the presence of rectal neoplasm, it will be interesting to observe whether the motility pattern change involves the whole colon or only that of the left side.

It has been pointed out that radical resection of the rectum for cancer simultaneously excises the parasympathetic nerves to the left colon. When the inferior mesenteric artery is also divided on the aorta, the sympathetic nerves to the same length of gut are also sectioned, as well as any of the occasional parasympathetic fibres which reach the gut with these sympathetic nerves. While the enteric plexus of the remaining left colon is still in continuity with that of the extrinsically innervated midtransverse colon, the descending colon which was the site of study was some $50 \mathrm{~cm}$ from the assumed last source of extrinsic vagal parasympathetic and sympathetic influence. Although therefore it is not possible to prove that the section of gut studied received no extrinsic nervous autonomic influence via the intrinsic plexus, this seems likely. Furthermore, because the mechanism by which a carcinoma of the rectum may disturb motility in the descending colon - if indeed it does so at this level - it not known, the effect of its excision is also speculative. These reservations must be kept in mind in trying to interpret the observations of this study.

Preoperatively there was clear evidence of a reduction of total colonic motility during sleep which 
has been previously described ${ }^{67}$ On waking, patients showed an immediate onset of vigorous activity (Fig. 1a). When breakfast was brought to the patients (Fig. 1b) an immediate increase of activity was again seen before the patient had even begun to eat. Snape ${ }^{8}$ has provided evidence that the increase in colonic motility on eating is initially by a neural mechanism with a later hormonal response particularly after a fatty meal. He did not comment, however, on the effect of the arrival of food. In this study, the immediacy of the colonic response to both waking and the arrival of food suggests that a nervous mechanism is responsible.

In the postoperative part of this study, the effect of sleep was still to reduce left colonic total activity; the responses to waking (Fig. 1c) and the arrival of food (Fig. 1d) were minimal, and the sleeping and awake motility indices were significantly reduced by surgery. These observations suggest that the segments of colon studied were extrinsically denervated by surgery. If this was indeed so, the reduction in colonic activity during sleep cannot be explained by the withdrawal of an extrinsic nervous influence, as this had been already removed. The possibility therefore arises that there is some non-nervous motor influence on the colon which is withdrawn during sleep, and this could be hormonal. Candidate hormones for this stimulatory role are many and amongst them are the enkephalins which are present in the brain and gut and which interact with specific opiate receptors in the colon. ${ }^{910}$

The measurement of the maximum periods of colonic inactivity again suggests that surgery caused an increase in colonic inertia. The achievement of statistical significance (at the $p=0.01$ level) of the observations, although few in number, when the data from the two patients who retained their sympathetic nerve supply to the segment studies were omitted, may indicate that in those two patients, the sympathetic nerves carried some parasympathetic fibres with them. ' Alternatively, some fibres of the sympathetic nerves may have caused stimulation of the gut as they appear to do in the dog," although it is generally believed that the sympathetic supply to the colon is inhibitory.

BOWEL MOTILITY AND DEFECATION

The apparent paradoxical associations of increased colonic motility with constipation and of reduced motility with diarrhoea in many circumstances are now well recognised. ${ }^{12}{ }^{13}$ In the first study series here no correlation could be made between bowel habit and motility at the time of presentation in part owing to the small numbers of patients. There is very suggestive evidence in this investigation, however, that rectosigmoid resection reduces colonic activity in the remaining left colon. At the time of the second series of investigations, although patients were generally over the worst of their postoperative bowel frequency pattern, nevertheless they had not all returned to their habitual frequency. It is a clinical observation that some never do after rectosigmoid resection. It is tempting to relate these two observations - reduced motility and increased bowel frequency. It may be suggested that this association is the consequence of the division of the motor nerves to the left side of the colon, and to note that truncal vagotomy which denervates the right colon is also frequently associated with an increased frequency of bowel habit. Obviously many more patients need to be studied before and, if possible, on several occasions after surgery to correlate motility pattern with return towards their habitual bowel habit. Such a study might show increasing motility over the postoperative months, just as the contractility of the stomach returns following truncal vagotomy. How this comes about remains to be understood.

\section{POSSIBLE IMPLICATIONS}

Should left sided paraysmpathectomy be shown to reduce the motility index of the left colon permanently, the possibility of using the operation to prevent deterioration and perhaps improve the symptoms of left sided colonic diverticulosis in some patients is apparent. Further longer term studies will be required, however, before such surgery could be recommended. There is already suggestive evidence that segmental resection of the sigmoid colon, which would at least partly denervate the more proximal left colon, reduces the complications of any remaining diverticulous change in that area, diverticular disease tending to spread in an orad direction from the sigmoid colon. ${ }^{14} 15$

I am grateful to the surgeons of St Mark's Hospital, London, for permission to study their patients, and to colleagues for help in assembling the apparatus required; $\mathrm{Mr} \mathrm{R} \mathrm{J}$ Nicholls was most helpful in this regard. I am very grateful to Dr C Williams and his colleagues who positioned the recording catheters for me. The help provided by St Mark's staff is also much appreciated. The Statistical Consulting Group of The University of Western Australia gave considerable assistance in the analysis of the results. My thanks also go to the Medical Illustrations unit of the Faculty of Medicine, The University of Western Australia, for preparation of the photographs, and to Miss J I Scott for preparation of the manuscript. My greatest thanks go to the patients for their willingness to be studied when they were concerned about their forthcoming surgery, and for their agreement to return for a second study. 


\section{References}

1 Mitchell GAG. Anatomy of the autonomic nervous system. Edinburgh and London: E\&S Livingstone, 1953: 190.

2 Lannon J, Weller E. The parasympathetic supply of the distal colon. Br J Surg 1947; 34: 373-8.

3 Painter NS. The etiology of diverticulosis of the colon with special reference to the action of certain drugs on the behaviour of the colon. Ann R Coll Surg Engl 1964; 34: $98-118$.

4 Dinoso VP, Murthy SNS, Goldstein J, Rosner B. Basal motor activity of the distal colon: a reappraisal. Gastroenterology 1983; 85: 637-42.

5 Frexinos J, Bueno L, Fioramonti J. Diurnal changes in myoelectric spiking activity of the human colon. Gastroenterology 1985; 88: 1104-10.

6 Adler HF, Atkinson AJ, Ivy AC. A study of the motility of the human colon: an explanation of dysynergia of the colon, or of the 'unstable colon'. Am J Dig Dis 1941; 8: 197-202.

7 Narducci F, Bassotti G, Gaburri M, Morelli A. Twentyfour hour manometric recording of colonic motor activity in healthy man. Gut $1987 ; 28: 17-25$.

8 Snape WJ, Wright SH, Battle WM, Cohen S. The gastrocolic response: evidence for a neural mechanism. Gastroenterology 1979; 77: 1235-40.

9 Stacher G, Steinringer H, Schmierer G. Effects of the synthetic enkephalin analogue FK 33-824 on colonic motor activity in man. In: Christensen J, ed. Gastrointestinal Motility. New York: Raven Press, 1980: 443-50.

10 Bueno L, Fioramonti J, Hondé C, Fargeas MJ, Primi MP. Central and peripheral control of gastrointestinal and colonic motility by endogenous opiates in conscious dogs. Gastroenterology 1985; 88: 549-56.

11 Wells JA, Mercer TH, Gray JS, Ivy AC. The motor innervation of the colon. Am J Physiol 1942; 138: 83-93.

12 Waller SL, Misiewicz JJ. Colonic motility in constipation and diarrhoea. Scand J Gastroenterology 1972; 7: 93-6.

13 Connell AM. The motility of the pelvic colon. II. Paradoxical motility in diarrhoea and constipation. Gut $1961 ; 3$ : 342-8.

14 Leigh JE, Judd ES, Waugh JM. Diverticulitis of the colon: recurrence after apparently adequate segmental resection. Am J Surg 1962; 103: 51-4.

15 Wychulis AR, Beahrs OH, Judd ES. Surgical management of diverticulitis of the colon. Surg Clin North Am 1967; 47: 961-9. 